\title{
Differential expression of epithelial-mesenchymal transition and stem cell markers in intrinsic subtypes of breast cancer
}

\author{
Victoria Pomp ${ }^{1}$ - Cornelia $\mathrm{Leo}^{2} \cdot$ Andrea Mauracher $^{3} \cdot$ Dimitri Korol $^{4}$. \\ Wenjun $\mathrm{Guo}^{5} \cdot$ Zsuzsanna Varga $^{1}$
}

Received: 9 September 2015/Accepted: 6 October 2015/Published online: 14 October 2015

(C) Springer Science+Business Media New York 2015

\begin{abstract}
The transcription factors SLUG and SOX9 have been shown to define mammary stem cell state. Similarly, epithelial-mesenchymal transition (EMT) markers (E-Cadherin, mTOR) have been shown to play a role in tumor-progression and metastatic potential in breast cancer. Finally, SOX10 is known to be expressed in breast cancer as well. The overexpressions of EMT and stem cell markers have been shown to correlate with poor overall survival. In this study, we examined whether the expression of these markers correlates with intrinsic subtypes of breast cancer and whether there is a prognostic difference in their expression-profile. We analyzed 617 breast cancer samples from two tissue micro arrays. Breast cancer samples were categorized into three groups according to hormone receptor expression and HER2-status as Luminal A/B, HER2-positive, and triple negative subgroup. Immunohistochemical expressions of SLUG, SOX9, SOX10, E-Cadherin, and mTOR were semi-quantitatively analyzed using a two-tiered and three-tiered scoring system in which cytoplasmic and nuclear stains were considered. Strong
\end{abstract}

Zsuzsanna Varga

zsuzsanna.varga@usz.ch

1 Institute of Surgical Pathology, University Hospital Zurich, Schmelzbergstrasse 12, 8091 Zurich, Switzerland

2 Department of Gynecology, Cantonal Hospital Baden, Baden, Switzerland

3 University Children's Hospital Zurich, Zurich, Switzerland

4 Cancer Registry, University Hospital Zurich, Zurich, Switzerland

5 Ruth L and Davis S Gottesman Institute for Stem Cell Biology and Regenerative Medicine, Department of Cell Biology, Albert Einstein College of Medicine, Bronx, NY, USA nuclear expression of SLUG was observed preferentially in triple negative but not in Luminal A/B or HER2-positive cases (24 vs. 3 and $0 \%, p<0.001$ ). Loss of SOX9 in the nuclear stain was less frequent in triple negative than in Luminal A/B or HER2-positive cases (4 vs. 9 vs. $13 \%$, $p<0.001)$. Expression of nuclear SOX10 was lower in triple negative than in Luminal A/B and HER2-positive cases (67 vs.78 and $79 \%, p=0.012$ ). E-Cadherin loss was observed only in Luminal A/B tumors $(p=0.016)$, no difference in the mTOR expression was seen between any of the three groups. No correlation to conventional histopathological-parameters or stage could be established in our cohort. Our study shows an inversed preferential nuclear expression of SLUG, SOX10, and SOX9 in triple negative and non-triple negative cases. This information is important in understanding the biology of triple negative breast cancer, also in terms of future studies dealing with targeted therapies based on the alterations of EMT and stem cell markers.

Keywords Intrinsic subtypes - Breast cancer - Stem cells $\cdot$ Epithelial-mesenchymal transition

\section{Introduction}

The various intrinsic subtypes of breast cancer have been receiving increased attention in stratifying breast cancer, especially in terms of prognostic and predictive information to aid clinical decision making [4, 7]. Although prognosis and therapeutic options for breast cancer have considerably improved over the last decade, intrinsic subtypes, such as triple negative breast cancer (TNBC) still have a poor prognosis [4, 7]. Several recent studies have addressed the role of epithelial-mesenchymal transition 
(EMT) factors and stem cell markers in breast cancer, with special emphasis on correlation to intrinsic subtyping [6, 9, 13, 16, 23]. Recently, Guo et al. described SLUG and SOX 9 as being mammary stem cell markers [9]. A change in the activation status of this gene couple leads to a tumorigenic progression of beast cells and to inhibition of the metastatic process [2, 9]. Furthermore, these markers can induce epithelial to mesenchymal phenotype in patients with early breast cancer onset $[9,23]$. This is important as EMT has been described playing a role in the transition of cells to a stem cell phenotype, which then can cause drug resistance and tumor recurrence [16, 20, 23]. SLUG is part of the snail family (SNAI2) and acts as a transcriptional repressor, important in the regulation of transcription processes of various genes and therefore also of protein synthesis in cells $[9,21]$. SOX9 on the other hand is a highly mobile transcription factor that plays a pivotal role in EMT in cells during embryogenesis [3]. Other SOX genes, such as SOX10, along with SOX9, are located in the sex determine region $\mathrm{Y}$ (SRY) [9, 21, 23].

SOX9 and SOX 10 are nuclear transcription factors with a High Monility Group (HMG) DNA-binding domain [3, 6]. Originally, SOX10 was discovered as survival mediating factor for neural crest cells allowing their differentiation into melanocytes and glia cells [6, 13]. Recently, SOX10 has been found in TNBC as well as in salivary gland tumors, especially in cells with a myoepithelial differentiation [6, 13].

E-Cadherin is a transmembrane cell adhesions protein usually expressed in luminal breast cells with epithelial differentiation $[11,22]$. The loss of E-Cadherin has been considered as a step for cancer cells to acquire a mesenchymal status in order to enter the EMT [16]. SLUG ties into this as it is one of several transcription factors involved in EMT that can downregulate the E-Cadherin expression [11]. The clinical importance of these factors was described by Choi et al. [5]. They found a significantly higher rate of EMT expression as well as a loss of E-Cadherin in invasive basal-like breast cancer [5].

The protein mTOR plays an important role in cell metabolism as well as in tumor development and growth and constitutes one part of the mammalian target of the Rapamycin (mTOR)/phosphoinositide 3 kinase (PI3K) pathway [19, 25]. Resistance to endocrine therapy has been attributed to the PI3K/mTOR pathway in estrogen receptor positive $(\mathrm{ER}+)$ breast cancer caused by an alternative activation of the usually hormone-dependent pathway [19, 25]. Furthermore, an active PI3K/mTOR pathway has been described to mediate resistance to Trastuzumab in HER2positive breast cancer. The mesenchymal-like subtype in the TNBC group seems to benefit from the activation of this pathway due to its responsiveness to drugs targeting mTOR [25].
In our study, we addressed the question, whether EMT and stem cell markers are differentially expressed in the intrinsic subtypes of breast cancer and what prognostic information these expression profiles have.

We systematically analyzed the above described panel of EMT and stem cell markers in a large cohort of breast cancer samples. These were divided into the intrinsic breast cancer subtypes as Luminal A/B, HER2-positive, and TNBC. We analyzed the protein expression of these markers using semiquantitative immunohistochemical scores and linked the scores to the intrinsic phenotype as well as to three traditional clinico-pathological parameters as grading, staging, and overall survival.

\section{Materials and methods}

\section{Patient cohort}

Altogether 617 breast cancer patients were included in this study. Tissue cores from archived formalin-fixed in paraffin-embedded tumors blocks containing invasive carcinoma from two tissue micro arrays (TMA) were used for the study. The cohort encompassed samples from the Institute of Surgical Pathology of the University Hospital Zurich between 1991 and 2011. Primary tumor tissue was available in $n=565$ cases, tumor tissue from recurrent lesions in $n=45$ cases, and tissue from lymph node metastasis in $n=7$ cases. All patients underwent either a mastectomy or a segmentectomy.

All clinico-pathological data and most of the data on patient survival were collected from the database of the Institute of Surgical Pathology, University Hospital Zurich. Follow-up information was additionally available through the Cancer Registry of the Canton Zurich.

This study is a part of a larger breast cancer study, which was previously approved by the Ethical Committee of the Canton Zürich (KEK-ZH NR: 2012-0553) and also by the internal review board of the Institute of Surgical Pathology, University Hospital Zurich.

\section{Definition of intrinsic subtypes}

The patients were grouped into three molecular subtypes based on definition in the literature as follows [26, 27]:

1. Triple negative phenotype (TNBC): All tumors with a negative estrogen and progesterone receptor status $(<1 \%)$ and negative HER 2 status (assessed either by immunohistochemistry and/or fluorescent in situ hybridization (FISH)).

2. Luminal A/B: All tumors with positive estrogen receptor $(>1 \%)$ and positive/or negative progesterone receptor status and positive/or negative HER2 status. 
3. HER2 positive tumors: All tumors with a positive HER2 Status (either being scored $3+$ by immunohistochemistry and/or amplified by FISH) and a negative hormone receptor status.

The hormone receptor and HER2 status of the invasive tumor tissue was taken from the original pathology reports. In total, we included $n=120$ triple negative, $n=457$ Luminal $\mathrm{A} / \mathrm{B}$, and $n=40$ HER2positive cases in this study.

\section{Clinic-pathological parameters}

All clinico-pathological parameters are shown in Tables 1, 2.
The age of our patient cohort ranged from 21 to 91 years of age. The lowest mean age was found in the HER 2 group with 53.9 years followed by the TN group with 55.5 years. The highest mean age was found in the Luminal $\mathrm{A} / \mathrm{B}$ Group with 58.5 years.

In total, primary tumor tissue was available from 565 patient (TN $n=116$, Luminal A/B $n=415$, HER2 positive $n=34)$, tissue from a local recurrence was available in 45 cases (TN $n=4$, Luminal A/B $n=37$, HER2-positive $n=4$ ), and axillary lymph node metastasis were available in 7 cases (TN $n=0$, Luminal A/B $n=5$, HER2-positive $n=2$ ).

Altogether $n=454$ tumors were diagnosed as invasive ductal carcinomas (TN $n=93$, Luminal A/B $n=325$,
Table 1 Clinical-pathological parameters of the breast cancer cohort, stratified according to intrinsic subtype

\begin{tabular}{|c|c|c|c|c|c|c|}
\hline \multirow[t]{3}{*}{$n=617$} & \multirow{2}{*}{\multicolumn{2}{|c|}{$\frac{\mathrm{TNBC}}{120}$}} & \multicolumn{2}{|c|}{ Luminal $\mathrm{A}$ and $\mathrm{B}$} & \multicolumn{2}{|c|}{ HER2 positive } \\
\hline & & & \multicolumn{2}{|l|}{457} & \multicolumn{2}{|l|}{40} \\
\hline & $n$ & $\%$ & $n$ & $\%$ & $n$ & $\%$ \\
\hline \multicolumn{7}{|l|}{ Tissue } \\
\hline Primary tumor & 116 & 97 & 415 & 91 & 34 & 85 \\
\hline Tumor recurrence & 4 & 3 & 37 & 8 & 4 & 10 \\
\hline Lymphnode metastasis & 0 & 0 & 5 & 1 & 2 & 5 \\
\hline \multicolumn{7}{|l|}{ Histological subtype } \\
\hline Invasive ductal & 93 & 78 & 325 & 71 & 36 & 90 \\
\hline Invasive lobular & 5 & 4 & 64 & 14 & 0 & 0 \\
\hline Other & 19 & 16 & 47 & 10 & 3 & 8 \\
\hline Not known & 3 & 2 & 21 & 5 & 1 & 2 \\
\hline \multicolumn{7}{|l|}{ Grade } \\
\hline G1 & 0 & 0 & 81 & 18 & 2 & 5 \\
\hline G2 & 13 & 11 & 244 & 53 & 7 & 18 \\
\hline G3 & 104 & 87 & 107 & 23 & 30 & 75 \\
\hline Not known & 3 & 2 & 25 & 5 & 1 & 2 \\
\hline \multicolumn{7}{|l|}{ Stage: pT } \\
\hline pT1 & 40 & 33 & 184 & 40 & 19 & 48 \\
\hline pT2 & 63 & 54 & 197 & 43 & 13 & 33 \\
\hline pT3 & 9 & 7 & 33 & 7 & 5 & 12 \\
\hline pT4 & 5 & 4 & 22 & 5 & 2 & 5 \\
\hline Not known & 3 & 2 & 21 & 5 & 1 & 2 \\
\hline \multicolumn{7}{|l|}{ Stage: pN } \\
\hline pNO & 57 & 47 & 139 & 30 & 15 & 38 \\
\hline $\mathrm{pN} 1$ & 35 & 29 & 195 & 43 & 15 & 38 \\
\hline $\mathrm{pN} 2$ & 14 & 12 & 35 & 8 & 1 & 2 \\
\hline $\mathrm{pN} 3$ & 7 & 6 & 22 & 5 & 6 & 15 \\
\hline Not known & 7 & 6 & 66 & 14 & 3 & 7 \\
\hline \multicolumn{7}{|l|}{ Stage M } \\
\hline M0 & 57 & 48 & 398 & 87 & 30 & 75 \\
\hline M1 & 28 & 23 & 14 & 3 & 4 & 10 \\
\hline Not known & 35 & 29 & 45 & 10 & 6 & 15 \\
\hline
\end{tabular}

$T N B C$ triple negative breast cancer 
Table 2 Age of the patients stratified according to intrinsic subtypes of breast cancer

\begin{tabular}{llll}
\hline & TNBC & Luminal A and B & HER2 positive \\
\hline Age (in years) & & & \\
Mean & 55.5 & 58.5 & 53.9 \\
Median & 54.5 & 59.0 & 53.0 \\
Minimum & 27 & 28 & 22 \\
Maximum & 88 & 91 & 87 \\
Not known & 0 & 127 & 11 \\
\hline
\end{tabular}

$T N B C$ triple negative breast cancer

HER2 positive $n=36$ ) and $n=69$ carcinomas were classified as an invasive lobular subtype (TN $n=5$, Luminal A/B $n=64$, HER2 positive $n=$ zero). Special subtypes such as medullary carcinoma, secretory carcinoma, and others were diagnosed in a separate category, which contained $n=69$ cases (TN $n=19$, Luminal A/B $n=47$, HER2 positive $n=3$ ). This information was deduced from the pathological diagnosis report and was missing from the database for 22 patients.

Grading was available in $n=588$ cases according to the modified Bloom and Richard grading score. 241 of 588 cases were poorly differentiated (G3: TN $n=104$, Luminal A/B $n=107$, HER 2 positive $n=30$ ), 264 of 588 cases were moderately differentiated (G2: TN $n=13$, Luminal A/B $n=244$, HER2 positive $n=7$ ), and 83 of 588 cases were well differentiated (G1: TN $n=0$, Luminal A/B $n=81$, HER2 positive $n=2$ ).

\section{Tissue Microarrays}

Two tissue microarrays were used in this study. The first tissue micro array (TMA 21) contained 544 single spots from all three subtypes, which were collected from 1991 to 2004. One spot per patient tissue sample was represented in this TMA. The second tissue micro array (TMA 174) contained 73 TNBC cases in double cores from the years 2005 to 2011.

The method of constructing these TMA-s from archived formalin-fixed, paraffin-embedded tumor blocks have been previously described [14, 24].

\section{Immunohistochemistry}

\section{E-Cadherin}

The clone EP700Y (Cell Marque Lifescreen Nr 246R-16) was used. Dilution 1:2000, with pretreatment with CC1 for $40 \mathrm{~min}$ and visualized with the OptiView kit from Ventana on the Ventana autosstainer. $m T O R$

The clone 7C10 (CellSignaling Nr 2983) was used. Dilution: 1:50, pretreatment $\mathrm{H} 2$ for $45 \mathrm{~min}$, visualized by the Refine HRP kit on the Leica/Zeiss Bond autostainer.

\section{SLUG}

The clone SLUG Klon C19G7 (Cell Signaling Nrl 9585) was used. Dilution 1:50, pretreatment in CC1 for $60 \mathrm{~min}$, visualized by ChromoMapDAB + UltraMap Rabbit on the Ventana autostainer.

SOX9

The clone Sox9 Polyklonal (Millipore Nr. AB5535) was used. Dilution: 1:4000, pretreatment $\mathrm{CC} 1$ for $48 \mathrm{~min}$, visualized with the OptiView kit from Ventana on the Ventana autostainer.

SOX10

The clone BC34 (Biocare Medical Nr. ACI3099) was used, Dilution 1:150, pretreatment $\mathrm{H} 2$ in $20 \mathrm{~min}$, visualized by the Refine HRP kit on the Leica/Zeiss Bons stainer.

\section{Scoring of immunohistochemical stains}

We used a two-tiered (negative vs. positive) and a threetiered (negative, mildly positive meaning $<50 \%$ of the cells are positive, and strongly positive meaning $>50 \%$ of the cells positive) scoring system.

The expressions in tumor cells of E-Cadherin and mTOR were scored with a two-tired system (negative vs. positive).

The nuclear expressions of SLUG, SOX 9, and SOX 10 in tumor cells were scored with a three-tired system. The cytoplasmic reaction of SLUG and SOX 10 was determined with a two-tiered system.

Illustrative photographs are shown in Figs. 1 and 2.

\section{Statistical analysis}

IBM SPSS Statistics 20 was used for the database and for the frequency evaluation in order to compare the TNBC group to the other two subtype groups. The frequency distribution probability was calculated by two-tailed Fisher's exact test with $2 \times 3$ or $2 \times 2$ contingency tables. Kaplan-Meier curves were used for a correlation analysis of markers and 5-year overall survival. Statistical significance was evaluated by $\log$ rank test. Overall, a $p$ value of under 0.05 was determined as being statistically significant. 

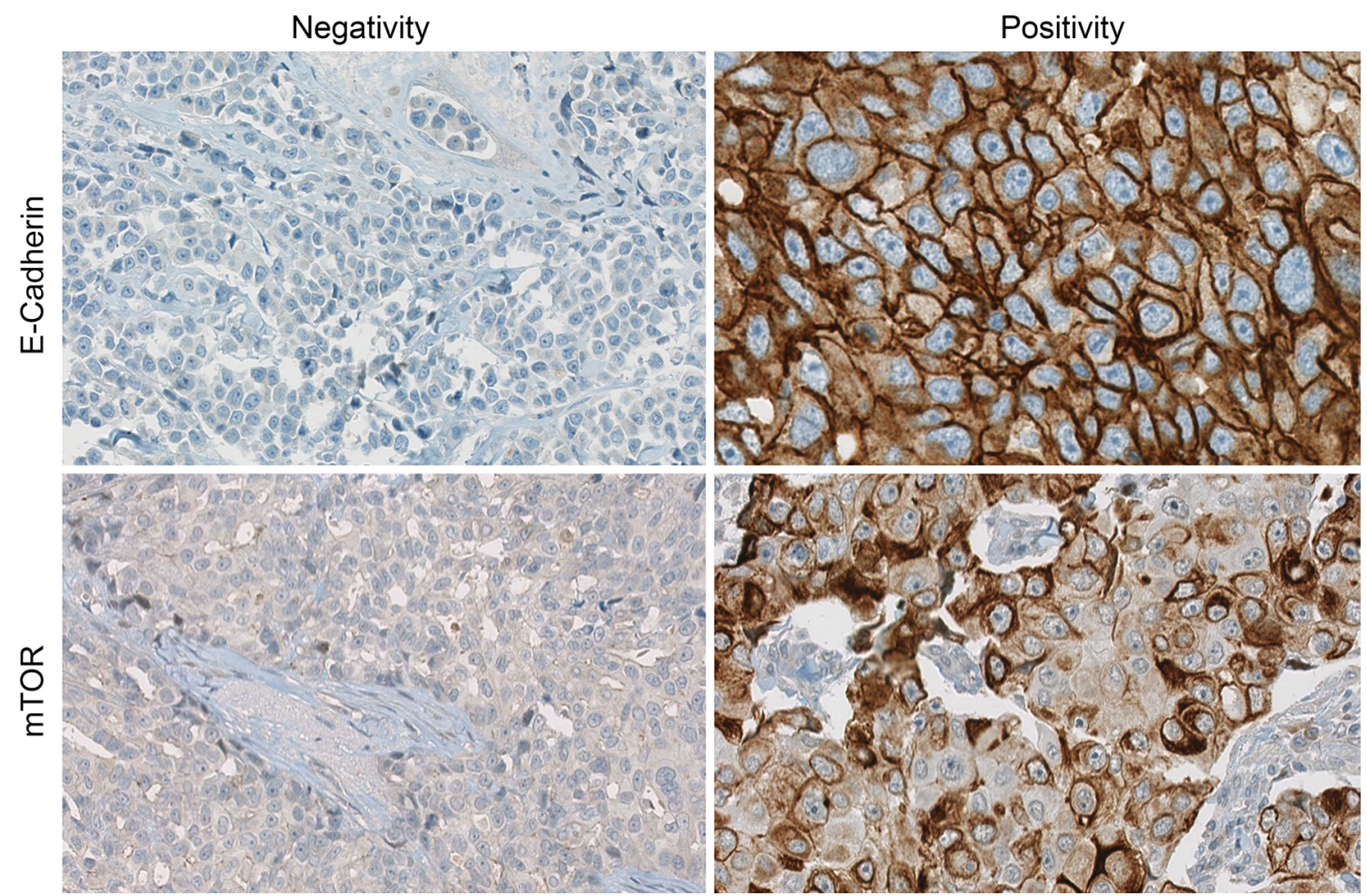

Fig. 1 Immunohistochemical expressions of E-Cadherin and mTOR in tissue microarrays scored with a two-tiered system for transmembrane expression

\section{Results}

\section{Nuclear expressions of SLUG, SOX9, and SOX10}

SLUG showed a differential nuclear expression in the three subtypes. In the TNBC group, we found $24 \%$ strongly positive, $40 \%$ mildly positive and $36 \%$ negative cases.

Strongly positive cases were seen in $3 \%$ of Luminal $\mathrm{A} / \mathrm{B}$ and in $0 \%$ in HER $2+$ positive cases. Mildly positive scored cases were seen in $49 \%$ of Luminal A/B and $45 \%$ of HER2 positive group. Negative stains were seen in $55 \%$ of HER2-positive and $48 \%$ of Luminal A/B cases.

Upon comparison of all three groups, the differences in the strong nuclear stain were statistically significant $(p<0.001)$.

SOX 9 expression was different in all three subtypes. A strong nuclear expression was seen in $88 \%$ of TNBC, in $65 \%$ of Luminal A/B and $67 \%$ of HER2 positive tumors. Mild nuclear stain was seen in $8 \%$ of TNBC, $26 \%$ of Luminal A/B cases, and $20 \%$ of HER 2 positive cases. A lack of expression was found in $4 \%$ of TNBC cases, $9 \%$ of Luminal A/B, and $13 \%$ of HER 2 positive cases.

The differences within the strong nuclear stains were statistically significant between the three groups (TN vs. Luminal A/B $p<0.001, \quad \mathrm{TN}$ vs. HER2-positive $p=0.011)$.
SOX10 was expressed differently in the three groups. Strong nuclear expression was common in the Luminal A/B $(78 \%)$ and the HER2-positive groups (79 \%) than in the TNBC $(67 \%)$ cases. Mild positivity was more common in TNBC (31\%) than in Luminal A/B (21\%) and HER2 positive $(14 \%)$ cases. Negative stains were found in $7 \%$ of HER2 positive cases, in $2 \%$ of TNBC and in $1 \%$ Luminal A/B tumors. A significant difference was found $(p=0.038)$ when comparing TNBC to Luminal $\mathrm{A} / \mathrm{B}$ subgroup. On the other hand, difference in protein expression between TNBC and HER2-positive groups was nearly significant $(p=0.06)$. Finally, comparing all three groups, protein expression was significantly different $(p=0.012)$.

\section{Cytoplasmic Reaction of SLUG and SOX 10}

The SLUG cytoplasmic stain was equal in all three subtypes: $94 \%$ of TNBC and of Luminal A/B, and $95 \%$ of HER2 positive cases expressed cytoplasmic SLUG (no significant difference).

SOX 10 cytoplasmic positivity was similar in all three groups: $97 \%$ of TNBC cases, $96 \%$ of HER 2 positive cases, and $93 \%$ of Luminal A/B cases (no significant difference). 

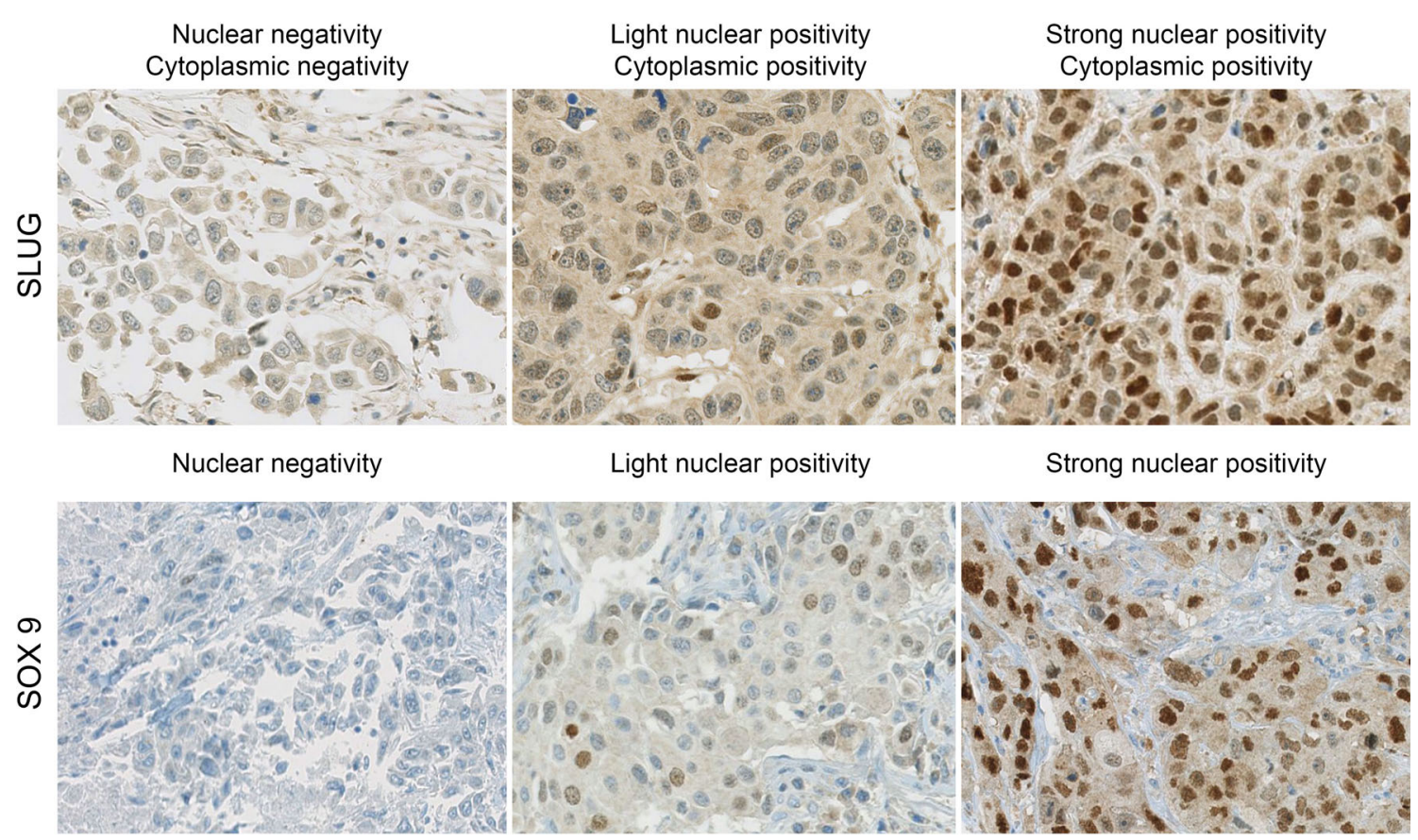

Light nuclear positivity

Strong nuclear positivity
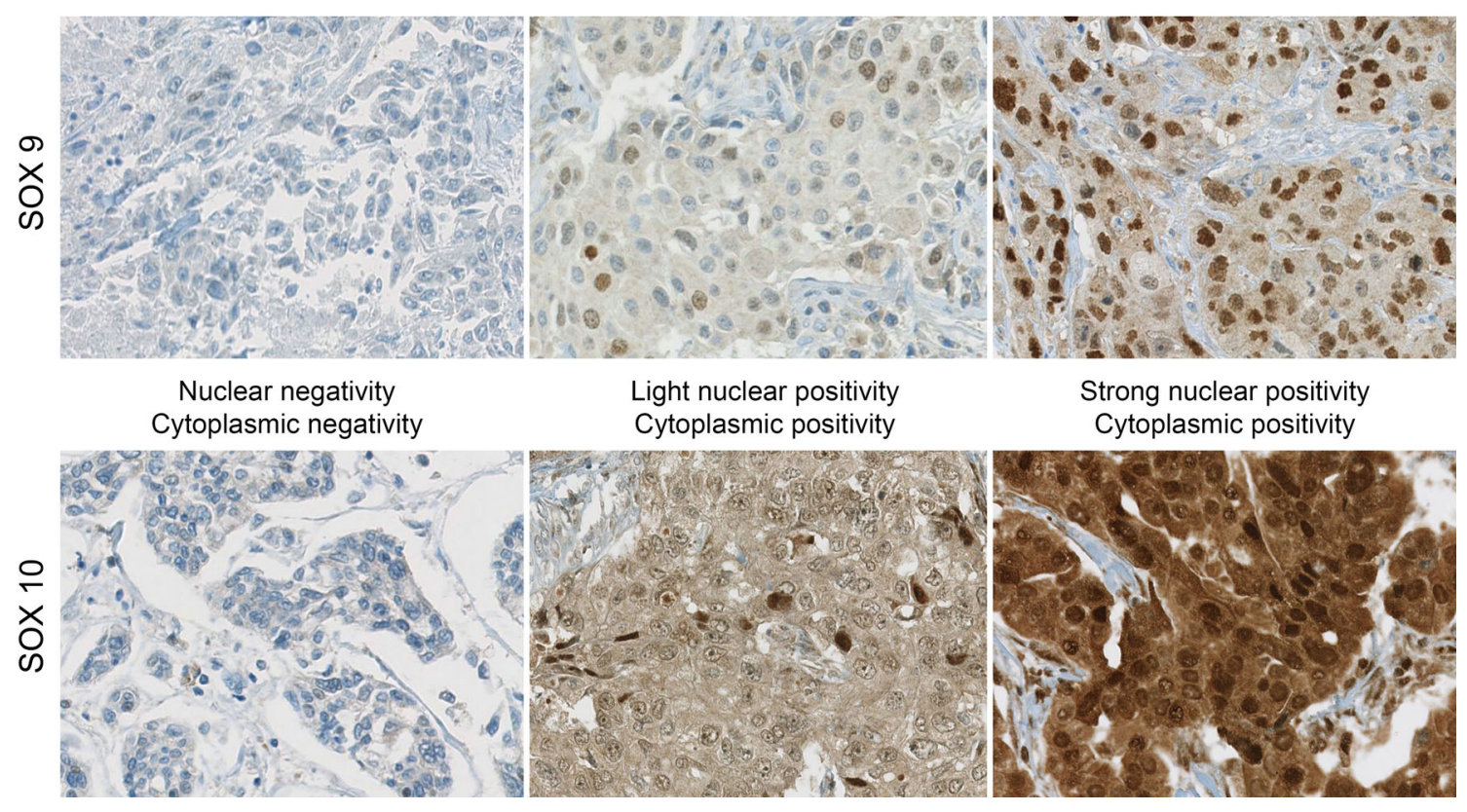

Light nuclear positivity Cytoplasmic positivity

Strong nuclear positivity Cytoplasmic positivity

Fig. 2 Immunohistochemical expressions of SLUG, SOX9, and SOX10 in tissue microarrays scored with a three-tired system for nuclear expression. Cytoplasmic expression was assessed of SLUG and SOX10 with a two-tiered scoring system

\section{E-Cadherin expression}

E-Cadherin positivity was more frequent in TNBC (114 of 120 cases) $(95 \%)$ than in Luminal A/B subgroup (378 of 439 cases) $(86 \%)$, this difference was statistically significant $(p=0.006)$.

No difference in E-Cadherin expression was found between the TNBC and HER2 positive groups $(p=0.26)$.

\section{mTOR expression}

mTOR was similarly expressed in all three subgroups: $78 \%$ of TNBC and Luminal A/B and $74 \%$ of HER2positive cases expressed mTOR ( $p=0.76$ comparing all three subgroups).

Results are shown in detail in Tables 3 and 4 and in Figs. 3 and 4.

\section{Correlation of E-Cadherin, mTOR, SLUG, SOX9, SOX10 expressions and 5-year overall survival}

The correlation of overall survival with the EMT and stem cell markers was quantified using Kaplan-Meier curves in all three subgroups. No significant correlation could be seen in any of the analyzed markers. The 5-year survival was evaluated in the TNBC subgroup as well but no significant correlation was found there either.

Results are shown in detail in Table 5.

\section{Correlation of E-Cadherin, mTOR, SLUG, SOX9, SOX10 expressions and histological grade and tumor stage}

SOX10 positive cytoplasmic expression correlated with the histological grade (G3) in the HER2-positive group (Pearson Chi-square $p=0.001$ ). 
Table 3 Expressions of EMT and stem cell markers in a two-tiered scoring system

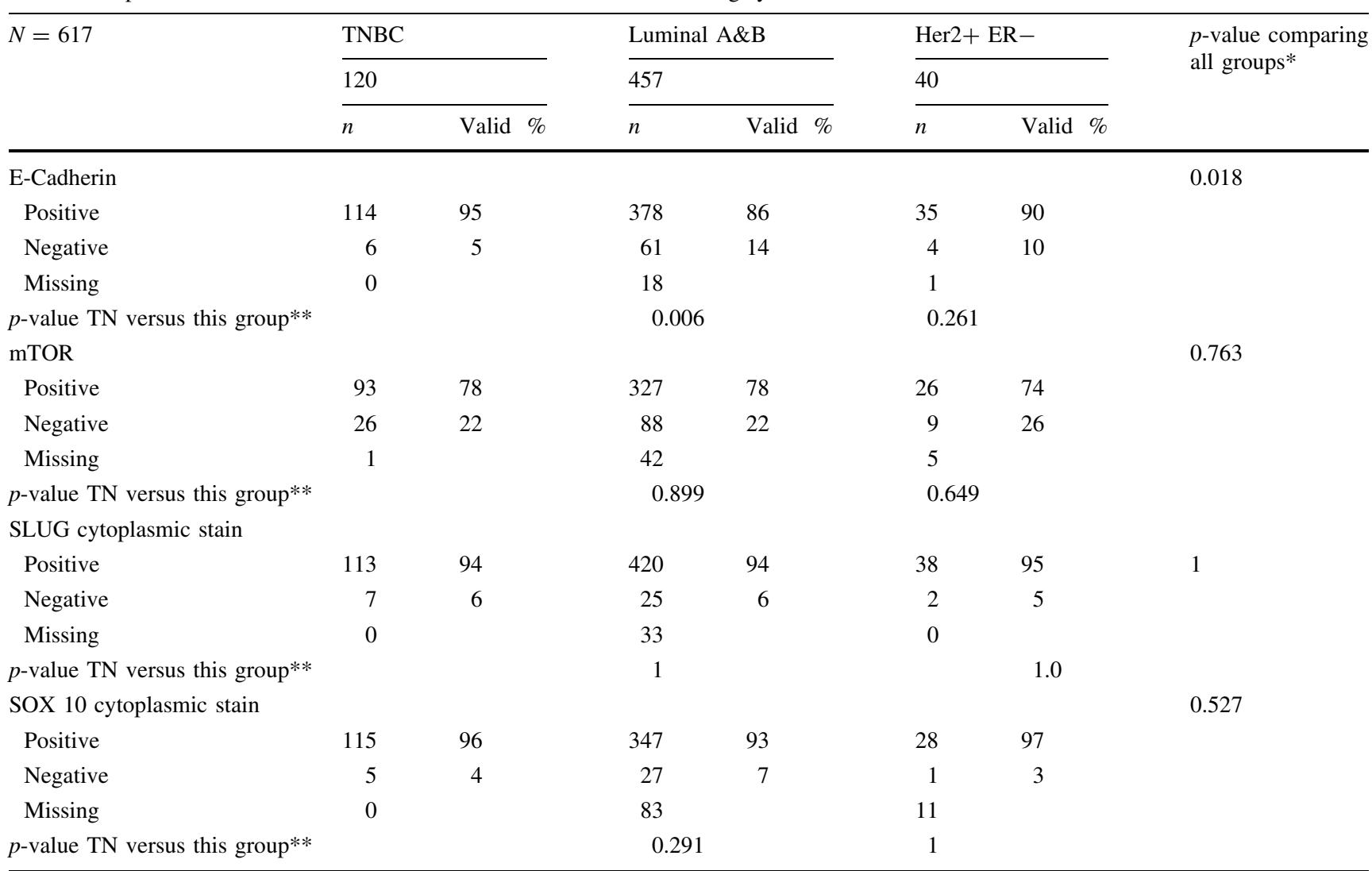

* Two-tailed Fisher's exact test with $2 \times 3$ contingency tables to calculate frequency distribution probability between all 3 groups

** Two-tailed Fisher's exact test with $2 \times 2$ contingency tables to calculate frequency distribution probability between TN and one other Subgroup

SLUG nuclear expression similarly correlated with the histological grade (G3) in the HER2-positive group (Pearson Chi-square $p=0.023$ ) as well as in the Luminal A/B group (Pearson Chi-square $p=0.008$ ).

SLUG cytoplasmic stain correlated with tumor stage (pT2) in the triple negative group (Pearson Chi-square $p=0.004$ ).

SOX9 nuclear expression correlated with the histological grade $(\mathrm{G} 2 / 3)$ in the Luminal A/B group (Linear association $p=0.035)$ and with tumor stage (pT2) in the triple negative group (Pearson Chi-square $p=0.019$ ).

No correlation between the nodal stage and the intrinsic subtype could be detected.

\section{Discussion}

In our study, we compared the protein expression profiles of EMT and stem cell markers in a cohort of breast cancer samples and looked for a correlation between expression profiles in TNBC, Luminal A/B, and HER2 positive intrinsic subgroups. We could show that EMT and stem cell markers are differentially expressed in these three intrinsic subtypes of invasive breast cancer. The TNBC intrinsic phenotype significantly differed in nuclear SLUG, SOX10, and SOX9 expressions from the Luminal A/B and HER2-positive subgroups. Preserved nuclear SLUG and nuclear SOX9 expressions as well as loss of nuclear SOX10 was a more common finding in TNBC, when comparing expression profiles with Luminal $\mathrm{A} / \mathrm{B}$ and HER2-positive groups. The Luminal A/B subgroup significantly differed from TNBC and HER2-positive cases with regards to the loss of E-Cadherin, while mTOR expression did not differ in the three intrinsic subtypes of our cohort.

Markers defining EMT and the stem cell phenotype in breast cancer have increasingly been the focus of attention in breast cancer research, as these factors have been described to influence tumor growth as well as metastatic properties of breast cancer [9, 17, 18]. Inhibition of EMT factors as an approach in the targeted therapies in TNBC has become an active field of basic research $[8,12]$. The fact that a strong nuclear SLUG is virtually missing in the HER2-positive cases of our cohort, needs further validation. In a recent study, a strong correlation between HER2- 
Table 4 Expressions of EMT and stem cell markers in three-tiered scoring system

\begin{tabular}{|c|c|c|c|c|c|c|c|}
\hline \multirow[t]{3}{*}{$N=617$} & \multicolumn{2}{|c|}{ TNBC } & \multicolumn{2}{|c|}{ Luminal $\mathrm{A} / \mathrm{B}$} & \multicolumn{2}{|c|}{ Her2+ ER- } & \multirow{3}{*}{$\begin{array}{l}p \text {-value comparing } \\
\text { all groups* }\end{array}$} \\
\hline & \multicolumn{2}{|l|}{120} & \multicolumn{2}{|l|}{457} & \multicolumn{2}{|l|}{40} & \\
\hline & $n$ & Valid \% & $n$ & Valid \% & $n$ & Valid $\%$ & \\
\hline SLUG nuclear stain & & & & & & & $<0.0001$ \\
\hline Strong positive & 29 & 24 & 13 & 3 & 0 & 0 & \\
\hline Light positive & 48 & 40 & 220 & 49 & 18 & 45 & \\
\hline Negative & 43 & 36 & 212 & 48 & 22 & 55 & \\
\hline Missing & 0 & & 12 & & 0 & & \\
\hline$p$-value $\mathrm{TN}$ versus this group** & & & 0.000 & & 0.0003 & & \\
\hline SOX 9 nuclear stain & & & & & & & $<0.0001$ \\
\hline Strong positive & 105 & 88 & 289 & 65 & 26 & 67 & \\
\hline Light positive & 10 & 8 & 118 & 26 & 8 & 20 & \\
\hline Negative & 5 & 4 & 39 & 9 & 5 & 13 & \\
\hline Missing & 0 & & 11 & & 1 & & \\
\hline$p$-value $\mathrm{TN}$ versus this group** & & & 0.000 & & 0.011 & & \\
\hline SOX 10 nuclear stain & & & & & & & 0.012 \\
\hline Strong positive & 81 & 67 & 294 & 78 & 23 & 79 & \\
\hline Light positive & 37 & 31 & 78 & 21 & 4 & 14 & \\
\hline Negative & 2 & 2 & 3 & 1 & 2 & 7 & \\
\hline Missing & 0 & & 82 & & 11 & & \\
\hline$p$-value $\mathrm{TN}$ versus this group** & & & 0.038 & & 0.060 & & \\
\hline
\end{tabular}

* Two-tailed Fisher's exact test with $3 \times 3$ contingency tables to calculate frequency distribution probability between all 3 groups

** Two-tailed Fisher's exact test with $2 \times 3$ contingency tables to calculate frequency distribution probability between TN and one other Subgroup

Fig. 3 Nuclear expressions of SLUG, SOX9, and SOX10 in a three-tiered scoring system (scores are shown in percentages)

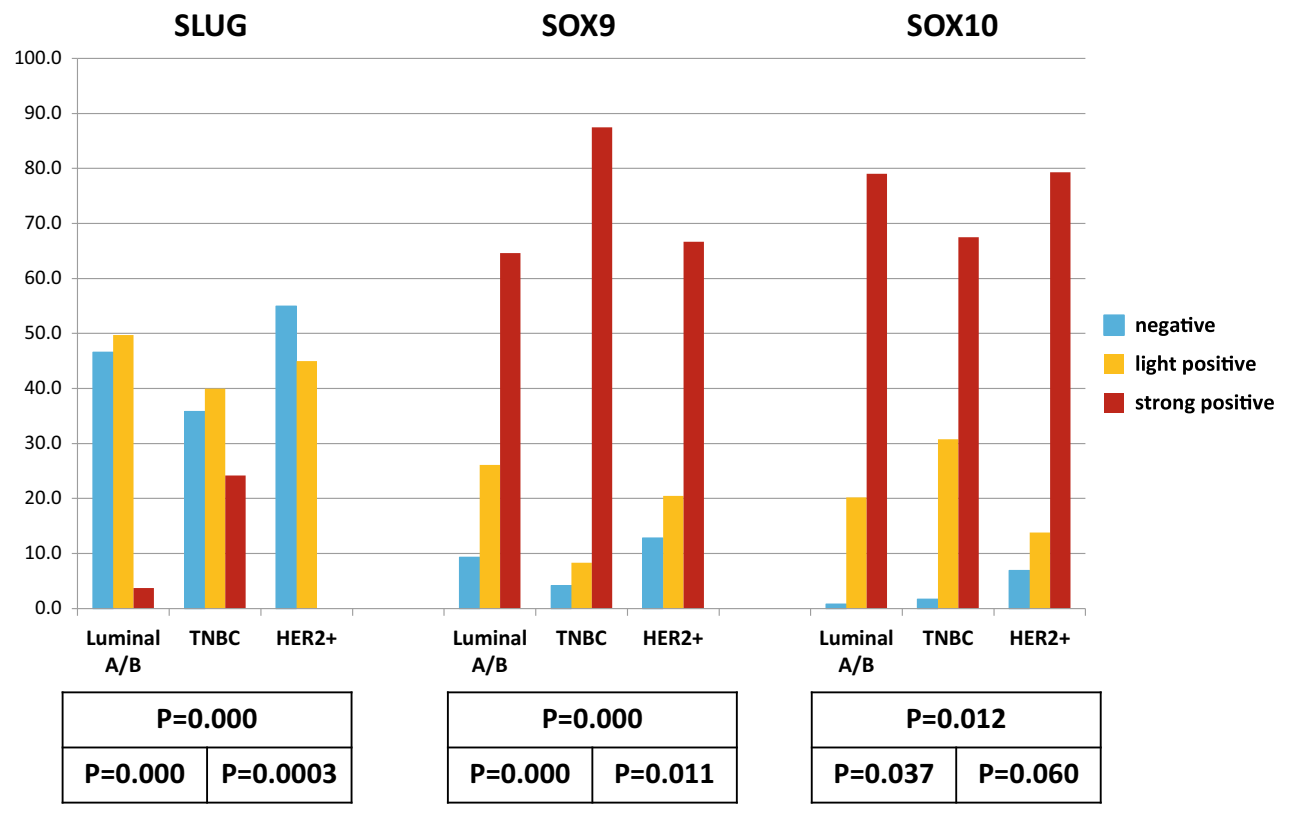

positive cell lines and induction of an EMT status was reported [10]. Furthermore, drug resistance in a SLUG positive cell line via binding to the ER promoter was also recently described [15].
Strongly maintained nuclear SLUG expression as a main finding in TNBC but not in Luminal A/B and HER2-positive samples corresponds well to what has been described in literature $[9,17,18,21]$. A recent work of Ito et al., 
Fig. 4 Expressions of E.Cadherin and mTOR in a twotiered scoring system (scores are shown in percentages)

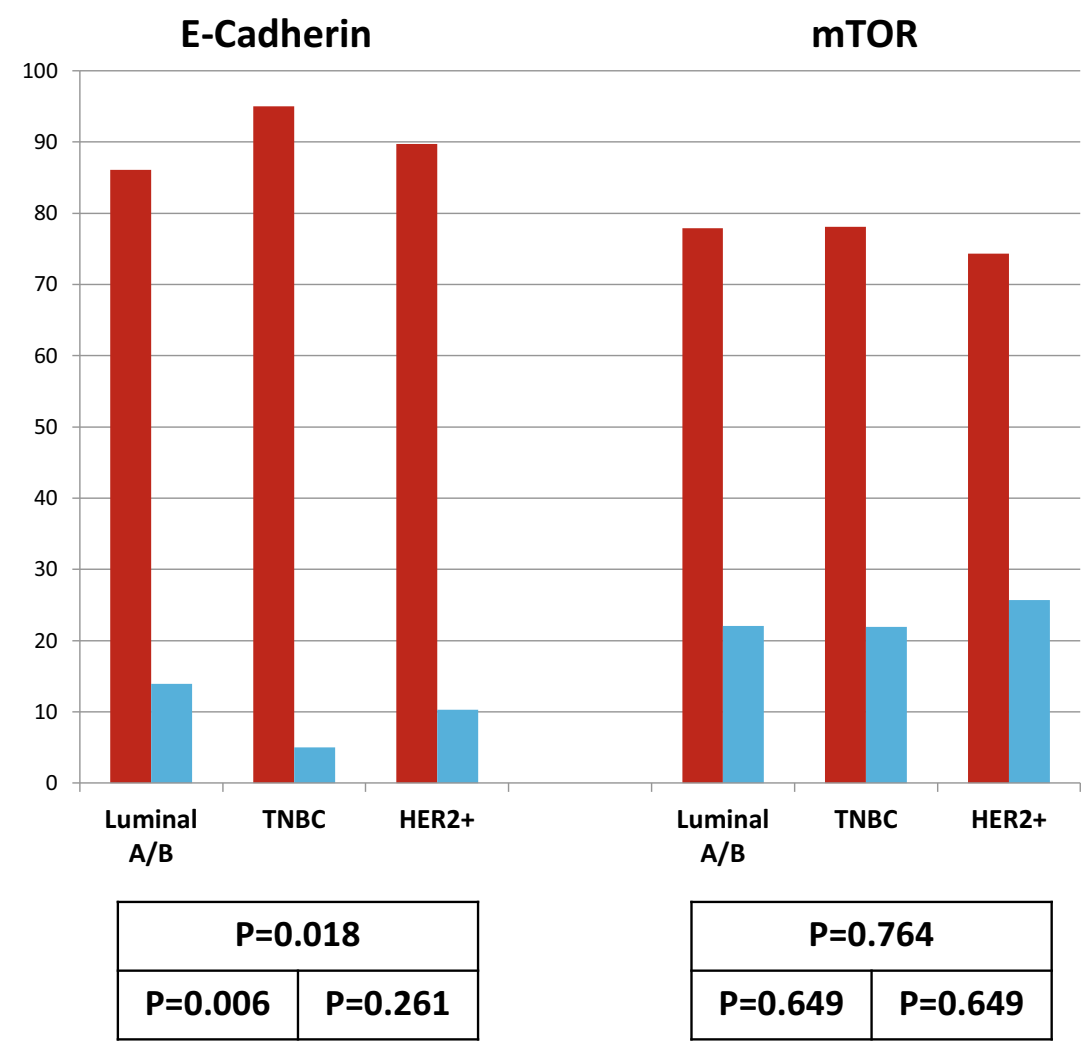

positive

negative
Table 5 Results of KaplanMeier curves Log Rank (Mantel-Cox) significant test in overall survival and in 5-year survival in terms of expressions of EMT and stem cell markers

\begin{tabular}{llllll}
\hline & E-Cadherin & mTor & Slug nuclear & SOX 9 & SOX 10 \\
\hline Overall survival & & & & & \\
TNBC & 0.541 & 0.752 & 0.682 & 0.311 & 0.620 \\
Luminal A\&B & 0.784 & 0.387 & 0.404 & 0.142 & 0.575 \\
HER2+ & 0.659 & 0.785 & 0.529 & 0.292 & 0.677 \\
5-year survival & & & & & \\
TNBC & 0.373 & 0.779 & 0.676 & 0.319 & 0.565 \\
\hline
\end{tabular}

regarding expression of stem cell markers including SLUG could not be linked to a poor prognosis, however SLUG expression together with one further transcription factor, ALDH1 was associated with shorter disease-free survival [12]. Similarly to our own observation, one further study by Alkatout et al., found no correlation between SLUG or other respective EMT markers and clinical outcome [1].

Higher levels of cytoplasmic SOX9 expression have been described as being present more commonly in TNBC than in other subtypes and a correlation of this expression with higher histological grade and poorer survival has also been reported $[3,9,21]$. We obtained similar results as we found the nuclear positivity of SOX9 to be more strongly positive in TNBC than in other subtypes, TNBC cases being mainly G3 in our cohort. The correlation of SOX9 expression to higher histological grade could be also confirmed in LumA/B cases in our cohort [3]. Nevertheless, no prognostic value was found in the nuclear/or cytoplasmic expression of SOX9 in our cohort when looking at overall or 5-year survival.

SOX10 nuclear expression was independently found to be more frequent in TNBC and basal-like breast cancer in two earlier studies $[6,13]$. Our study showed that strong nuclear expression of SOX10 was similarly present in TNBC, Luminal A/B, and HER2-positive cases. However there was a significant difference in the detection of mild positivity or loss of a nuclear stain, this was more frequently detected in the HER2 positive group than in the TNBC or Luminal A/B cases of our cohort. Our results further support the hypothesis, that SOX10 expression is a common finding in cancer exhibiting myoepithelial and basal differentiation [6, 13]. Nevertheless, the biological importance of the loss in SOX10 expression in HER2 positive breast cancer is currently not fully understood.

Differences to previous studies regarding the lack of an association between markers and overall survival in our 
study can be partially explained by possible intra-tumoral heterogeneity. The fact, that two tissue cores were available in the TNBC cases, and only one tissue core from the other groups in our study, can be considered as one further factor in increasing intra-tumoral heterogeneity.

E-Cadherin, as a hall-mark in the process of EMT was almost equally present in the TNBC and HER 2 positive group, providing no further prognostic information on these subgroups in our cohort. This result contradicts earlier findings where the loss of E-Cadherin was more commonly detected in the invasive basal-like phenotype [5]. The increased loss of E-Cadherin in the Luminal A/B subgroup of our study can be explained by the higher frequency of an invasive lobular phenotype in this group, typically being hormone receptor positive than of a basallike phenotype.

The expression of mTOR, which is a part of the PIK3K/ Akt/mTOR pathway and has been well established to influence tumor growth, could not be differentiated using the the intrinsic subtypes in our study [19]. This result corresponds to the described presence of the mTOR pathway in all there subtypes by Vicient et al. [25]. mTOR inhibitors, such as Everolimus, are being considered in the treatment of hormone receptor positive and HER2-negative and metastatic or locally advanced breast cancer [19].

\section{Conclusion}

Our data show evidence, that stem cell and EMT markers are differentially expressed in the basic intrinsic subtypes of breast cancer, being preferentially preserved in TNBC. Despite lack of correlation to prognosis in our study, this data may serve as additional information in evaluating targeted therapies based on the alterations of EMT and stem cell markers. Further studies will be needed to identify the exact prognostic role of EMT and stem cell markers, when stratifying breast cancer according to intrinsic subtypes.

\begin{abstract}
Acknowledgments The authors wish to thank to Mrs. Martina Storz for preparing the TMA sections, to Mr André Fitsche for performing all immunohistochemical stains and to $\mathrm{Mr}$. André Wethmar and $\mathrm{Mr}$ Norbert Wey for preparing the high quality illustrations and performing the digitalization of the stained TMA slides.
\end{abstract}

\section{Compliance with ethical standards}

Conflict of interest The authors have no conflict of interest to disclose.

\section{References}

1. Alkatout I, Wiedermann M, Bauer M, Wenners A, Jonat W, Klapper W (2013) Transcription factors associated with epithelial-mesenchymal transition and cancer stem cells in the tumor centre and margin of invasive breast cancer. Exp Mol Pathol 94:168-173. doi:10.1016/j.yexmp.2012.09.003

2. Anwar TE, Kleer CG (2013) Tissue-based identification of stem cells and epithelial-to-mesenchymal transition in breast cancer. Hum Pathol 44:1457-1464. doi:10.1016/j.humpath. 2013.01.005

3. Chakravarty G, Moroz K, Makridakis NM, Lloyd SA, Galvez SE, Canavello PR, Lacey MR, Agrawal K, Mondal D (2011) Prognostic significance of cytoplasmic SOX9 in invasive ductal carcinoma and metastatic breast cancer. Exp Biol Med (Maywood) 236:145-155. doi:10.1258/ebm.2010.010086236/2/145

4. Cheang MC, Voduc D, Bajdik C, Leung S, McKinney S, Chia SK, Perou CM, Nielsen TO (2008) Basal-like breast cancer defined by five biomarkers has superior prognostic value than triple-negative phenotype. Clin Cancer Res 14:1368-1376. doi:10.1158/1078-0432.CCR-07-1658

5. Choi Y, Lee HJ, Jang MH, Gwak JM, Lee KS, Kim EJ, Kim HJ, Lee HE, Park SY (2013) Epithelial-mesenchymal transition increases during the progression of in situ to invasive basal-like breast cancer. Hum Pathol 44:2581-2589. doi:10.1016/j.hum path.2013.07.003

6. Cimino-Mathews A, Subhawong AP, Elwood H, Warzecha HN, Sharma R, Park BH, Taube JM, Illei PB, Argani P (2013) Neural crest transcription factor Sox 10 is preferentially expressed in triple-negative and metaplastic breast carcinomas. Hum Pathol 44:959-965. doi:10.1016/j.humpath.2012.09.005

7. Goldhirsch A, Winer EP, Coates AS, Gelber RD, Piccart-Gebhart M, Thurlimann B, Senn HJ, Panel Members (2013) Personalizing the treatment of women with early breast cancer: highlights of the St Gallen International Expert Consensus on the Primary Therapy of Early Breast Cancer 2013. Ann Oncol 24:2206-2223. doi:10. 1093/annonc/mdt303

8. Granados-Principal S, Liu Y, Guevara ML, Blanco E, Choi DS, Qian W, Patel T, Rodriguez AA, Cusimano J, Weiss HL, Zhao H, Landis MD, Dave B, Gross SS, Chang JC (2015) Inhibition of iNOS as a novel effective targeted therapy against triple-negative breast cancer. Breast Cancer Res 17:25. doi:10.1186/s13058-015$0527-\mathrm{x}$

9. Guo W, Keckesova Z, Donaher JL, Shibue T, Tischler V, Reinhardt F, Itzkovitz S, Noske A, Zurrer-Hardi U, Bell G, Tam WL, Mani SA, van Oudenaarden A, Weinberg RA (2012) Slug and Sox9 cooperatively determine the mammary stem cell state. Cell 148:1015-1028. doi:10.1016/j.cell.2012.02.008S0092-8674(12)00165-1

10. Gupta P, Srivastava SK (2014) Inhibition of Integrin-HER2 signaling by Cucurbitacin $\mathrm{B}$ leads to in vitro and in vivo breast tumor growth suppression. Oncotarget 5:1812-1828

11. Hugo HJ, Kokkinos MI, Blick T, Ackland ML, Thompson EW, Newgreen DF (2011) Defining the E-cadherin repressor interactome in epithelial-mesenchymal transition: the PMC42 model as a case study. Cells Tissues Organs 193:23-40. doi:10.1159/ 000320174

12. Ito $M$, Shien $T$, Omori M, Mizoo $T$, Iwamoto $T$, Nogami $T$, Motoki T, Taira N, Doihara H, Miyoshi S (2015) Evaluation of aldehyde dehydrogenase 1 and transcription factors in both primary breast cancer and axillary lymph node metastases as a prognostic factor. Breast Cancer. doi:10.1007/s12282-015-0583-1

13. Ivanov SV, Panaccione A, Nonaka D, Prasad ML, Boyd KL, Brown B, Guo Y, Sewell A, Yarbrough WG (2013) Diagnostic SOX10 gene signatures in salivary adenoid cystic and breast basal-like carcinomas. Br J Cancer 109:444-451. doi:10.1038/ bjc.2013.326bjc2013326

14. Kononen J, Bubendorf L, Kallioniemi A, Bärlund M, Schraml P, Leighton S, Torhorst J, Mihatsch MJ, Sauter G, Kallioniemi OP (1998) Tissue microarrays for high-throughput molecular profiling of tumor specimens. Nat Med 4:844-847 
15. Li Y, Wu Y, Abbatiello TC, Wu WL, Kim JR, Sarkissyan M, Sarkissyan S, Chung SS, Elshimali Y, Vadgama JV (2015) Slug contributes to cancer progression by direct regulation of ERalpha signaling pathway. Int J Oncol 46:1461-1472. doi:10.3892/ijo. 2015.2878

16. Mallini P, Lennard T, Kirby J, Meeson A (2014) Epithelial-tomesenchymal transition: what is the impact on breast cancer stem cells and drug resistance. Cancer Treat Rev 40:341-348. doi:10. 1016/j.ctrv.2013.09.008

17. Markiewicz A, Ahrends T, Welnicka-Jaskiewicz M, Seroczynska B, Skokowski J, Jaskiewicz J, Szade J, Biernat W, Zaczek AJ (2012) Expression of epithelial to mesenchymal transition-related markers in lymph node metastases as a surrogate for primary tumor metastatic potential in breast cancer. J Transl Med 10:226. doi:10.1186/1479-5876-10-2261479-5876-10-226

18. Mego M, Mani SA, Lee BN, Li C, Evans KW, Cohen EN, Gao H, Jackson SA, Giordano A, Hortobagyi GN, Cristofanilli M, Lucci A, Reuben JM (2012) Expression of epithelial-mesenchymal transition-inducing transcription factors in primary breast cancer: the effect of neoadjuvant therapy. Int $\mathrm{J}$ Cancer 130:808-816. doi:10.1002/ijc. 26037

19. Paplomata E, O'Regan R (2014) The PI3K/AKT/mTOR pathway in breast cancer: targets, trials and biomarkers. Ther Adv Med Oncol 6:154-166. doi:10.1177/1758834014530023

20. Reya T, Morrison SJ, Clarke MF, Weissman IL (2001) Stem cells, cancer, and cancer stem cells. Nature 414:105-111. doi:10. $1038 / 35102167$

21. Riemenschnitter C, Teleki I, Tischler V, Guo W, Varga Z (2013) Stability and prognostic value of Slug, Sox 9 and Sox 10 expression in breast cancers treated with neoadjuvant chemotherapy. Springerplus 2:695. doi:10.1186/2193-1801-2-695

22. Shamir ER, Ewald AJ (2015) Adhesion in mammary development: novel roles for E-cadherin in individual and collective cell migration. Curr Top Dev Biol 112:353-382. doi:10.1016/bs.ctdb. 2014.12.001

23. Soady K, Smalley MJ (2012) Slugging their way to immortality: driving mammary epithelial cells into a stem cell-like state. Breast Cancer Res 14:319

24. Theurillat JP, Zürrer-Härdi U, Varga Z, Storz M, Probst-Hensch NM, Seifert B, Fehr MK, Fink D, Ferrone S, Pestalozzi B, Jungbluth AA, Chen YT, Jäger D, Knuth A, Moch H (2007) NYBR-1 protein expression in breast carcinoma: a mammary gland differentiation antigen as target for cancer immunotherapy. Cancer Immunol Immunother 56:1723-1731. doi:10.1007/ s00262-007-0316-1

25. Vicier C, Dieci MV, Arnedos M, Delaloge S, Viens P, Andre F (2014) Clinical development of mTOR inhibitors in breast cancer. Breast Cancer Res 16:203. doi:10.1186/bcr3618

26. Weigelt B, Geyer FC, Reis-Filho JS (2010) Histological types of breast cancer: how special are they? Mol Oncol 4:192-208. doi:10.1016/j.molonc.2010.04.004

27. Weigelt B, Mackay A, A'Hern R, Natrajan R, Tan DS, Dowsett M, Ashworth A, Reis-Filho JS (2010) Breast cancer molecular profiling with single sample predictors: a retrospective analysis. Lancet Oncol 11:339-349. doi:10.1016/S1470-2045(10) 70008-5 